\title{
Respuestas metabólicas y fisiológicas de tres métodos de entrenamiento de fuerza en mujeres entrenadas.
}

\section{Metabolic and physiological responses of three methods of strength training in trained women}

\author{
Tenerife Daniel ${ }^{1}$, Arakaki Nicollas ${ }^{1}$, Santos Diego ${ }^{1}$ Emmanuel de Medeiros \\ Lima Leonardo ${ }^{2}$, Miguel Enrique ${ }^{3}$, Marsili Guedes Krom ${ }^{3}$, Correia Rocha \\ Alexandre $^{5}$, Guedes Jr Dilmar ${ }^{6}$, Pereira da Silva Rodrigo ${ }^{7}$. \\ ${ }^{1}$ Universidade Metropolitana de Santos \\ ${ }^{2}$ Univeridade Metodista de Piracicaba \\ ${ }^{3}$ Centro Universitário de Espirito Santo do Pinhal \\ ${ }^{4}$ Universidade Metropolitana de Santos, Universidade Federal de São Paulo \\ ${ }^{5}$ Universidade Metropolitana de Santos, Universidade Paulista \\ ${ }^{6}$ Universidade Metropolitana de Santos, Centro de Estudos de Fisiologia do Exercício e \\ Treinamento, Universidade Santa Cecilia \\ ${ }^{7}$ Universidade Metropolitana de Santos, Faculdade Praia Grande, Universidade Federal \\ de São Paulo
}

\section{Resumen}

Objetivo: Analizar las respuestas metabólicas y fisiológicas de los métodos de repetición regresiva alterna, series fijas bilaterales y series fijas unilaterales en mujeres entrenadas.

Metodología: La muestra incluye 10 mujeres con una edad promedio de $32.1 \pm 3.7$ años, que han estado practicando entrenamiento de fuerza por más de 4 años. Cada voluntario asistió al laboratorio durante 4 días, el primer día para realizar la prueba 1RM y los otros días para realizar métodos alternativos de repetición regresiva (RRA), series fijas bilaterales (SFB) y series fijas unilaterales (SFU) al azar. El tiempo total, las repeticiones realizadas, el lactato en sangre, la frecuencia cardíaca máxima, la percepción del dolor y el esfuerzo se midieron utilizando las escalas Borg y Omni.

Resultados: Hubo una diferencia significativa $(p<0.01)$ en las variables de repetición entre los métodos: SFB $-41.4 \pm 9.40$; SFU $-79.1 \pm 13.3$ y RRA $-72.0 \pm 0.0$. y tiempo total, RRA con 114 segundos, 77 y 150 segundos de diferencia para SFB y SFU respectivamente. En los otros análisis, no se observaron diferencias significativas $(\mathrm{p}>0.05)$.

Conclusión: No hubo diferencias significativas en las escalas de lactato sanguíneo, volumen total, Borg y Omni entre los métodos, sin embargo, hubo una diferencia significativa en la variable de tiempo, que fue menor para el método de repetición regresiva alternativa en relación con los demás.

Palabras clave: Entrenamiento de fuerza, lactato, métodos de culturismo

\section{Abstract}

Objective: Analyze the acute effects of the alternate repeat, bilateral fixed series and unilateral fixed series methods in trained women.

Methodology: The research was composed by 10 female individuals with at least 4 years of bodybuilding practice. Each one of them did a 4 days try-out, which the first day evaluate the maximum load, and the other days concluded the alternating regressive repetition (RRA), bilateral fixed series (SFB) and unilateral fixed series (SFU). Total time, repetitions performed, blood lactate, maximum heart rate, pain perception and effort using the Borg and Omni scales were measured.

Results: There was a significant difference $(p<0,01)$ in the repetition between the methods: SFB - 41,4 \pm 9,40; SFU - 79,1 $\pm 13,3$ e RRA - 72,0 $\pm 0,0$. And total time

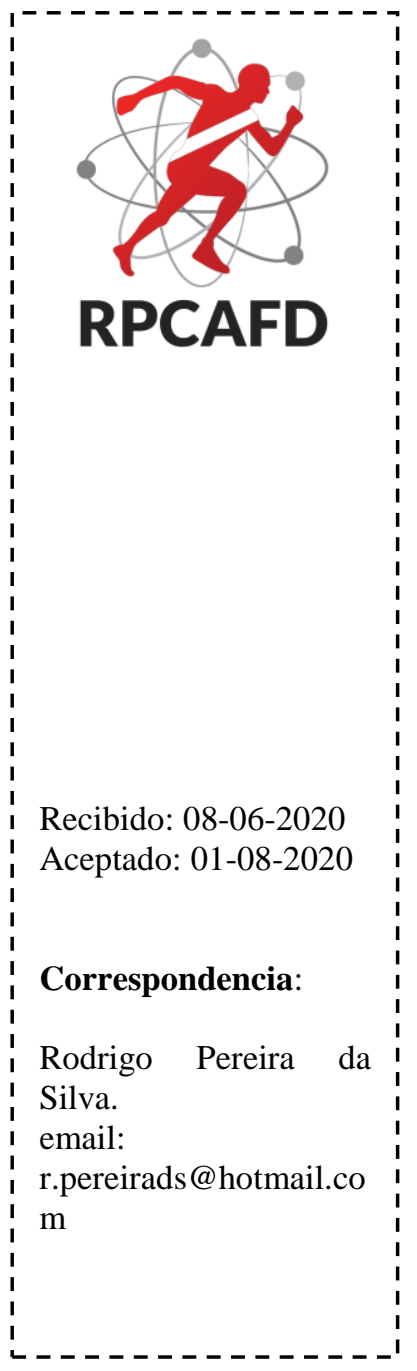


RRA with 114 seconds, 77 and 150 seconds difference for SFB and SFU respectively. In the other analyzes, no significant difference was observed between the methods.

Conclusion: The present research didn't find any significant difference in the blood lactate, volume, Borg and Omnis cales between the methods. However, there was a significant difference in the time variation of the alternating regressive repetition method for the others.

Keywords: Strenght training, Lactate, Bodybuilding methods.

\section{Introdução}

O treinamento de força (TF) consiste em exercícios que utilizam a contração voluntária da musculatura esquelética contra alguma forma de resistência que pode ser conseguida por meio do próprio corpo, pesos livres ou máquinas 1,2,3,4. O Treinamento de Força proporciona mudanças no desempenho motor, na força muscular, na composição corporal e, consequentemente, na estética corporal ${ }^{5}$, o mesmo tem um papel importante nos programas de atividade física e tem sido recomendado por organizações de saúde na intenção de beneficiar a saúde geral e condicionamento físico da população ${ }^{5}$. A prática do TF regular por mulheres pode diminuir a tensão e o estresse, melhorando a quantidade e a qualidade do sono $^{6,7}$. É visto que a mulher que pratica o TF obtém bons resultados em relação ao aumento de força e hipertrofia muscular, sendo que o ganho de força sobrepõe o ganho de hipertrofia 8,9 .

A resposta do lactato sanguíneo frente a diferentes métodos de TF tem sido alvo de investigação ${ }^{10}$. O lactato sanguíneo pode exercer grande influência nos processos metabólicos relacionados ao treinamento de alta intensidade, inclusive em relação à síntese e secreção de testosterona ${ }^{11}$. O acúmulo desta substância nos músculos pode causa dor e desconforto após o exercício ${ }^{12}$. Assim, o indicativo da concentração sanguínea do lactato permite avaliar indiretamente a acidose metabólica do exercício, sendo uma das ferramentas diagnósticas utilizadas pela fisiologia do exercício ${ }^{13}$. A concentração de lactato no sangue é de aproximadamente 1,0 $\mathrm{mmol} / \mathrm{L}$ a $1,8 \mathrm{mmol} / \mathrm{L}$, em repouso e durante o exercício leve, quando existe equilíbrio entre sua produção muscular e sua remoção hepática ${ }^{14}$. À medida que o exercício físico se intensifica, ocorre um desequilíbrio entre a produção e a remoção, com consequente acúmulo de lactato no sangue e aumento de sua concentração. A detecção desse índice de limitação funcional é feita com a realização de um teste com cargas crescentes e baseia-se na medida da concentração sanguínea do lactato, coletas seriadas de sangue são feitas para se determinar o limiar anaeróbio, que ocorre quando a concentração do lactato excede o valor de 4,0 mmol/L. O lactato está envolvido diretamente na sinalização para secreção de hormônio do crescimento (GH) e testosterona, posteriormente, de forma sistemática, gerando hipertrofia ${ }^{15}$.

Com o objetivo de potencializar a hipertrofia muscular foram desenvolvidos vários métodos que têm sido testados cientificamente ${ }^{16,17}$. Alguns estudos analisaram a reposta do lactato sanguíneo e outras variáveis fisiológicas mediante aos diferentes métodos de TF 18,19,20,21,22. Apesar de vários estudos investigarem as respostas e os ajustes fisiológicos de diferentes métodos de TF são escassos os que analisam a aplicação e os resultados desses métodos na população feminina.

Analisar as respostas metabólicas e fisiológicas dos métodos Repetição Regressiva Alternada (RRA), série fixa bilateral e série fixa unilateral em mulheres treinadas.

\section{Metodologia}

O tipo de estudo contemplou um delineamento experimental e transversal que foi composta por 10 mulheres, com idade entre 25 a 34 anos, residentes da cidade de SantosSP-Brasil, treinadas com no mínimo 4 anos de experiência de prática regular de TF.

\section{Técnicas e Procedimentos}

Inicialmente avaliamos a massa corporal total e a estatura utilizando um estadiômetro da marca Sanny®. Em seguida, as 
voluntarias realizaram o teste de carga máxima $(1 \mathrm{RM})^{1}$, no exercício cadeira extensora da marca Athetic $\AA$, seguindo o protocolo que consistiu no aquecimento de 5 a 10 repetições com intervalo de 1 minuto, 3 a 5 repetições, intervalo de 2 minutos, e a última série de 2 a 3 repetições, intervalo de 3 minutos. A execução do teste 1RM foi realizada com três tentativas respeitando intervalo de 3 minutos. A tentativa executada com a maior quantidade de peso, foi utilizada para análise. Os níveis de lactato sanguíneo foram mensurados na ponta dos dedos logo após o término dos métodos, utilizando aparelho da marca Lactate Analyzer (Accutrend). Monitoramos a frequência cardíaca durante as execuções dos métodos utilizando um frequencímetro da marca Polar ${ }^{\circledR}$ RS800. A circunferência da coxa foi aferida antes e imediatamente após a realização dos métodos utilizando uma fita métrica da marca Sanny®. Foram coletados o volume total (peso $\mathrm{x}$ reps $\mathrm{x}$ séries), o tempo total para a execução dos métodos, a frequência cardíaca máxima após o teste, e os níveis de esforço através das escalas subjetivas de dor ${ }^{23}$ e esforço Omni ${ }^{24,25}$.

Os critérios de inclusão adotados foram: mulheres com experiencia no treinamento com pesos, idade entre 20 a 40 anos e voluntariado para executar 4 dias de avaliações. Já os critérios de exclusão incluíram lesões musculoesqueléticas nos membros inferiores, desistência ou não realização de todas as sessões do estudo. Este estudo foi aprovado pelo comitê de ética e pesquisa da Universidade Metropolitana de Santos CAAE 12734919.0.0000.5509.

Cada voluntária compareceu ao laboratório em quatro ocasiões. No primeiro dia foi realizado o teste de $1 \mathrm{RM}$ para a prescrição da carga a ser utilizada para cada método e nos demais dias foram realizados os métodos analisados. Anteriormente a execução dos métodos, ases voluntarias realizavam um aquecimento de 3 séries de 10 a 12 repetições com $20 \%$ da carga máxima e com intervalo de 2 minutos. A ordem de realização dos métodos ocorreu de forma aleatória para cada voluntária e os métodos analisados foram: série fixa bilateral (SFB), utilizando $70 \%$ de 1RM, série fixa unilateral (SFU) com $35 \%$ de 1RM e Repetição Regressiva Alternada (RRA) executando a proposta com $35 \%$ de 1RM. O método RRA consiste em realizar de 8 até 1 repetição de forma alternada (direito e esquerdo) e sem pausa, sendo 36 reps para cada lado e totalizando 72 repetições. Pela escassez de estudos sobre o método não foi possível identificar a porcentagem de peso baseado na literatura, com isso foi realizado um estudo piloto para se chegar à porcentagem citada anteriormente. Os métodos SFB e SFU foram realizados em três séries até a falha concêntrica momentânea e com intervalo de 60 segundos entre as séries, e para o RRA foi realizada uma única série. $O$ intervalo entre a execução dos métodos foi de 72 horas.

\section{Estatística}

Inicialmente foi realizada uma análise descritiva dos dados com média e desvio padrão. Após a confirmação da não normalidade dos dados optou-se por utilizar o teste de medidas repetidas com post hoc em Bonferoni. O nível de significância aceito foi $\mathrm{p} \leq 0,05$.

\section{Resultados}

A tabela 1, apresenta as características antropométricas da amostra estudada. Os resultados apresentados na tabela 2 indicam diferença significativa nas repetições do método serie fixa bilateral para os demais métodos, porém se for levado em consideração às repetições de cada membro não houve diferença. Ainda analisando a mesma tabela, ocorreu uma disparidade significativa na variável tempo do método RRA para os demais.

Tabela 1: Idade, tempo de pratica de musculação sistemática, antropometria e teste de carga máxima na cadeira extensora das voluntarias analisadas.

\begin{tabular}{ccccc}
\hline Idade (anos) & Tempo de prática (anos) & Altura(m) & Peso (kg) & Teste 1RM(kg) \\
\hline $28 \pm 6,7$ & $6,9 \pm 4,3$ & $1,67 \pm 0,06$ & $70,3 \pm 6,6$ & $82,7 \pm 5,5$
\end{tabular}

Os dados estão em forma de média e desvio padrão ( \pm ). 
$\mathrm{Na}$ tabela 3 não foram encontradas diferenças nas variáveis de respostas fisiológicas, de circunferência e nas escalas subjetivas de controle de dor e esforço. Na tabela 2 os resultados referentes à repetição foram SFB de 41,9 $( \pm 9,40)$, SFU 79,1 $( \pm 13,3)$. O método RRA teve a pré programação para 36 repetições para cada membro, totalizando 72 repetições. Na variável repetição houve diferença significativa na série fixa bilateral para os demais métodos, más considerando a unilateralidade dos outros dois métodos não há disparidade.

Tabela 2: Repostas das repetições, tempo total e volume de treinamento entre os métodos avaliados.

\begin{tabular}{lllcc}
\hline Métodos & Peso $(\mathrm{kg})$ & Repetições & Tempo (segundos) & Volume \\
\hline SFB & $57,9 \pm 3,1$ & $41,4 \pm 9,40^{*}$ & $191 \pm 17$ & $2,397,1 \pm 882,6$ \\
SFU & $28,9 \pm 2,5$ & $79,1 \pm 13,3$ & $264 \pm 40$ & $2,285,9 \pm 661,2$ \\
RRA & $28,9 \pm 2,9$ & $72,0 \pm 0,0$ & $114 \pm 24^{*}$ & $2,080,8 \pm 208,7$ \\
\hline
\end{tabular}

* Indica diferença significativa entre os métodos ( $\mathrm{p} \leq 0,01)$; os dados estão em forma de média e desvio padrão $( \pm)$; série fixa bilateral (SFB); série fixa unilateral (SFU); regressão regressiva alternada (RRA).

Tabela 3: Repostas do lactato e da frequência cardíaca entre os métodos avaliados.

\begin{tabular}{lcccccl}
\hline Métodos & Lactato & Fcmáx & Cnf Pré & Cnf Pós & Borg & Omni \\
\hline SFB & $8,3 \pm 2,1$ & $161,4 \pm 6,5$ & $61,8 \pm 2,4$ & $62,8 \pm 2,4$ & $7,8 \pm 1,7$ & $9,4 \pm 0,9$ \\
SFU & $7,8 \pm 2,3$ & $161,9 \pm 6,7$ & $61,7 \pm 2,8$ & $63,0 \pm 3,1$ & $7,5 \pm 0,7$ & $9,5 \pm 0,8$ \\
RRA & $7,1 \pm 1,2$ & $163,9 \pm 4,5$ & $62,0 \pm 2,1$ & $62,8 \pm 2,4$ & $7,3 \pm 1,4$ & $9,2 \pm 0,6$
\end{tabular}

Os dados estão em forma de média e desvio padrão ( \pm ); lactato sanguíneo (lactato) em $\mathrm{mm} / \mathrm{mol}$; Fcmáx(frequência cardíaca máxima) em batimentos por minuto; circunferência da coxa (Cnf) avaliado em centímetros; Escala subjetiva de dor Borg (Borg) e esforço (Omni); série fixa bilateral (SFB); série fixa unilateral (SFU); repetição regressiva alternada (RRA).

\section{Discussão}

Encontramos diferenças significativas do método SFB comparado aos outros métodos no número de repetições máximas executadas. O método RRA foi executado em uma unidade tempo menor em relação aos outros analisados. Analisando o tempo total do exercício houve diferença significativa entre os métodos, SFB foi de 191 segundos. SFU deu-se 264 segundos. E o RRA realizou-se em 114 segundos. O método RRA demonstrou ter uma duração curta quando comparado aos outros métodos e mostrou ser benéfico em relação ao tempo eficiente. Resultados distintos foram observados por Guedes ${ }^{18}$, onde o método drop set apresentou diferença significativa na variável tempo de 339 segundos. Corroborando com nossos achados, o estudo citado à cima apresentou um tempo relativamente maior de duração quando comparado ao nosso, nos levando a crer que se pode utilizar um método de treinamento com um menor tempo de duração e ter respostas metabólicas semelhantes.

De acordo com a tabela 3 o lactato produzido no método SFB foi de $8,3 \mathrm{mmol} / \mathrm{L}(2,12$ $\mathrm{mmol} / \mathrm{L})$, SFU deu-se 7,8 mmol/L (2,29 mmol/L), e no RRA deu $7,16 \mathrm{mmol} / \mathrm{L}(1,23 \mathrm{mmol} / \mathrm{L})$. Não houve diferença significativa na amostra do lactato entre os métodos realizados. Guedes ${ }^{18}$ encontrou uma diferença na variável lactato entre os métodos, deu-se $9,1 \mathrm{mmol} / \mathrm{L}$ (1,9 mmol/L) no drop set (DS), já no método tradicional (TR) foi 7,2 $\mathrm{mmol} / \mathrm{L}(2,4$ $\mathrm{mmol} / \mathrm{L}$ ). Foram realizadas três séries para cada um dos métodos utilizados. O drop set foi realizado com cargas de $80 \%, 60 \%$ e $40 \%$ de 1RM, consecutivamente e intervalo entre séries de 60 
segundos e no método tradicional a carga foi de $70 \%$ e pausa de 60 segundos, ambos os métodos utilizaram a falha concêntrica momentânea cada série; uma hipótese para a semelhança no lactato do métodos SFB e TR é a utilização do mesmo protocolo de falha concêntrica, para a diferença da variável lactato do DS para os demais é a variável peso, a carga utilizada foi maior comparada ao do presente estudo. De acordo com Ahtiainen ${ }^{28}$ houve aumento de lactato em ambos os métodos, repetições máximas e repetições forçadas, mas não houve diferenças significativas entre as cargas. Outros resultados da literatura mostram que o lactato produzido no leg press horizontal pelos indivíduos $\mathrm{B}$ e C (mulheres) foram de $4,4 \mathrm{mmol} / \mathrm{L}(0,14 \mathrm{mmol} / \mathrm{L})$, utilizaram o protocolo de múltiplas séries sendo quatro séries com dez repetições para serem executadas a $70 \%$ (1RM) e intervalo de 40 segundos para cada série ${ }^{29}$. No presente estudo os resultados do lactato sanguíneo foram superiores em relação ao estudo mencionado; uma hipótese para essa diferença entre o lactato é que no presente estudo usou-se o protocolo de repetições até a falha concêntrica, outra hipótese é que antecedentemente os indivíduos $\mathrm{B}$ e $\mathrm{C}$ realizarem o método leg press haviam feito quatro séries de dez repetições à $70 \%$ (1RM) em outro exercício de membro superior. Cesar $^{30}$ analisou os efeitos fisiológicos de dois protocolos de treinamento com pesos, força máxima (Fmax) e resistência muscular localizada (RML), em jovens mulheres treinadas. $\mathrm{Na}$ análise de lactato houve diferença significativa entre os mesmos. O protocolo de RML apresentou maior densidade comparada ao Fmax, por apresentar uma concentração maior de lactato em menos tempo de treinamento ${ }^{31,32}$.

Os resultados dos estudos referenciados previamente não corroboram com o nosso pela duração distinta dos métodos e protocolos utilizados. No presente estudo, equalizamos métodos de treinamento que visam a hipertrofia ${ }^{33}$, já Cesar, et al. ${ }^{30}$ utilizaram o protocolo de treinamento visando gasto energético ${ }^{34}$. Nos resultados do estudo de Sobral \& Rocha ${ }^{19}$ constatou-se o aumento do lactato pós dois diferentes métodos de treinamento com um destaque ao protocolo pirâmide decrescente. O estudo de Guedes ${ }^{18}$ corrobora com os resultados obtidos em nosso estudo, onde se constatou o aumento do lactato sanguíneo após diferentes métodos de treinamento de força, não houve diferença significativa no nível de lactato sanguíneo nos métodos investigados, sendo assim apresentando resultados similares ao nosso, entretanto é importante ressaltar que ambos os estudos investigaram homens. Os resultados de outros estudos não corroboram com os nossos achados pela disparidade de protocolos utilizados; foram dois 2021,8(1): $1064-1072$ programas de TF com volumes e intensidades diferentes com distintos intervalos entre as séries 22,35 .

O estudo de Tiggemann ${ }^{26}$ que teve como objetivo de pesquisar sobre a percepção de esforço alega em seu estudo que o aumento da quantidade de repetições realizadas em determinado exercício ou sessão causa o aumento da percepção de esforço, atribuídos ao grau de fadiga nos músculos envolvidos. No estudo de Guedes ${ }^{18}$ onde teve como objetivo comparar respostas fisiológicas do treinamento de força realizou-se o método tradicional, que se assemelha ao método SFB realizado no presente estudo. $\mathrm{Na}$ variável repetição do método supradito efetuou-se $28,7( \pm 7,1)$ houve diferença significativa se comparado aos dos outros métodos do estudo em questão; e quando comparado ao presente estudo foi menor do que as repetições do método SFB; uma hipótese para o ocorrido é que o estudo de Guedes ${ }^{18}$ equalizou os métodos para membros superiores. No experimento de Nasser \& Neto ${ }^{27}$ observou-se que treinamento equalizado com cargas menos e altas repetições se mostram eficazes para hipertrofia muscular tanto de membros superiores quanto inferiores. O estudo supracitado não corrobora com nossos achados pelas cargas distintas.

Método de treinamento é a maneira pela qual se combinam as variáveis agudas de treinamento, a fim de proporcionar diferentes estímulos fisiológicos e motivacionais. Já um método avançado de treino é aquele que utiliza a mesma base conceitual, porém com objetivo primário de intensificar os estímulos em relação aos modelos tradicionais de treino, elevando sobretudo o volume, intensidade e a densidade do treino (Teixeira, 2015). A densidade variável que representa a quantidade de estímulos aplicados na unidade de tempo. Para calcular a densidade, devese dividir o produto de volume e intensidade pelo tempo de treino ([volume $\mathrm{X}$ intensidade]/tempo) ${ }^{18}$.

O estudo de Marshall 37 demostrou resultados significativos comparando o Rest Pause com modelo tradicional de treino usando a mesma sobrecarga externa. Os resultados demostraram aumento do sinal eletromiográfico dos músculos envolvidos no agachamento, uma das hipóteses que pode explicar os achados deste estudo é a densidade do Rest Pause. Outro estudo comparou os efeitos de três modelos de treino com volume total equalizado (drop set, múltiplas séries e pirâmide crescente) na área de secção transversa do vasto lateral de homens treinados. Após 12 semanas de intervenção, os resultados revelaram aumento significante nas três intervenções, sem diferenças entre elas. Cabe ressaltar que o método de séries descendentes 
atingiu o mesmo volume total de treino em menos tempo (estratégia time-efficient), elevando a densidade de treino ${ }^{38}$, corroborando com os nossos achados em relação ao método RRA.

As limitações estão sobre o número pequeno de mulheres avaliadas, entretanto a amostra é de voluntarias extremamente treinadas com pesos. No dia a dia, sugerimos a variação dos métodos entre os praticantes, entretanto se a falta de tempo for um empecilho, utilizar o método RRA.

$\mathrm{O}$ presente estudo não encontrou diferença significativa para o lactato sanguíneo, volume total, escalas subjetivas de Borg e Omni entre os métodos analisados. O método RRA se mostrou capaz de ser realizado em menor tempo que os demais métodos analisados, se mostrando uma proposta eficiente quando o objetivo é economia de tempo (time efficient), além de proporcionar alta densidade ao treinamento. Os resultados sugerem a elaboração de novos protocolos com amostras de ambos os sexos e estudos longitudinais para conclusões mais robustas.

\section{Referências:}

1. Guedes JR, DP; Rocha, AC; Teixeira, CVLS; Guedes, KM Silva, RP. Hipertrofia muscular: a ciência na pratica em academia. São Paulo: CREF4/SP, 2018.

2. ACSM. American College of Sports Medicine position stand. Progression models in resistance training for healthy adults. Medicine and Science in Sports and Exercise, Madison, 2009, v.41, n.3, p.364-380.

3. Silva, MS, Cortez, ACL, Dantas, EHM. Efeitos do treinamento de musculação nos níveis de potência de membros inferiores em mulheres adultas. Biomotriz, 2018, 12(3).

4. Marcos-Pardo PJ, Martínez-Rodríguez A, Gil-Arias A. Impact of a motivational resistancetraining programme on adherence and body composition in the elderly. Sci Rep. 2018;8(1):1370. doi:10.1038/s41598-018-19764-6

5. Kraemer WJ, Ratamess, N. A., Alvar, B. A., Evetoch, T. E., Housh, T. J., Ben Kibler, W., $\&$ Triplett, N. T. Progression models in resistance training for healthy adults. Medicine and science in sports and exercise, 2009, 1(3), 687-708.

6. Guedes JR, DP. Musculação estética e saúde feminina. São Paulo: Phote, 2003.

7. ACSM. American College of Sports Medicine. Quantity and quality of exercise for developing and maintaining cardiorespiratory, musculoskeletal, and neuromotor fitness in apparently healthy adults: guidance for prescribing exercise. position stand. Med Sci Sports Exerc. 2011; 43(7):1334-59.

8. Sartori, C. A.; Simeão Júnior, CA. Benefícios do treinamento de força para mulheres. Revista Digital. Buenos Aires, Anõ 18, n 185, Octubre de 2013.

9. Silva, DAS. Efeito do exercício intervalado na capacidade aeróbia, composição corporal e na população obesa: uma revisão baseada em evidências. Motriz, Rio Claro, 2013 v.16, n.2, 468-476, abr./jun.2013.

10. Takano, H., Morita, T., Iida, H., Asada, K., Kato, M., Uno, K., HIrose, K., Matsumoto, A., Takenaka, K., Hirata, Y., Eto, F., Nagai, R., sato, Y., \& nakajima, T. Hemodynamic and hormonal responses to a shortterm low-intensity resistance exercise with the reduction of muscle blood flow. European Journal of Applied Physiology, 2005 v. 95 p. 65-73.

11. Marques, A. T.; Baldissera, V. Identificação do limiar de lactato nos exercícios resistidos: rosca bíceps e mesa flexora. Revista Digital - Buenos Aires - 2005, Anõ 10. Agosto de 2005.

12. MCardle, W. Katch, W. Katch. Fisiologia do Exercício. Energia, Nutrição e Desempenho Humano. $7^{a}$ Edição. Rio de Janeiro, Guanabara Koogan, 2011

13. Pereira, B; Souza Junior, TP. Metabolismo celular e exercício físico: aspectos bioquímicos e nutricionais. Phorte Editora Ltda, 2010.

14. Wirtz, N., Wahl, P., Kleinöder, H., \& Mester, J. Lactate kinetics during multiple set resistance exercise. Journal of sports science \& medicine, 2014, 13.(1): 73-77. 
15. Reeves, G. V, Kraemer, R. R., Hollander, D. B., Clavier,J., Thomas, C., Francois, M., \& Castracane, V. D. Comparison of hormone responses following light resistance exercise with partialvascular occlusion and moderately difficult resistance exercise without occlusion. Journal ofapplied physiology, 2006, 101(6), 1616-1622.

16. Azevedo, P. H. S. M.; Oliveira, J. C.; Aguiar, A. P.; Oliveira, P. A. F.; Barros, C. L., Agostini, G. G., Garcia, E. S., \& Baldissera, V. Limiar de Lactato em Exercício Resistido. Revista Motriz, 2004, 10(1), 31 - 36

17. Mezzaroba, PV; Ribeiro, MS; Machado, FA. Comparação de dois métodos de treinamento contra resistência na força, antropometria e composição corporal de mulheres JOVENS Revista Brasileira de Ciência e Movimento, 2014, v. 22, n. 2, p. 106-113.

18. Guedes, KM; silva, RP; ScorcinE, C; Madureira, F; Guedes JR, DP. Comparação das respostas metabólicas e fisiológicas dos métodos Tradicional, Drop Set e Oclusão Vascular Parcial em indivíduos treinados. Simpósio Internacional de ciências do esporte, 2018.

19. Sobral, MC; Rocha, AC. Respostas do lactato sanguíneo e da dor muscular de inicio tardio pós dois métodos distintos de treinamento de força. Revista Brasileira de Prescrição e Fisiologia do Exercicio (RBPFEX), 2017, v. 11, n.66, p.284-292.

20. Scott, CB. Contribution of blood lactate tothe energy expenditure of weight training. Journal of Strength \& Conditioning Research, 2006, 20(2), 404-408.

21. Siqueira, LODC, Prado, MM, Simionato, AR, Sancassani, A, Pessôa Filho, D M. Acute response of blood lactate to different weight training protocols. Revista Brasileira de Medicina do Esporte, 2018, 24(1), 26-30.

22. González-Badillo, J. J., Rodríguez-Rosell, D., Sánchez-Medina, L., Gorostiaga, E. M., \& pareja-Blanco, F. "Maximal intended velocity training induces greater gains in bench press performance than deliberately slower half-velocity training." European journal of sport Science, 2014, 14.8: 772-781.

23. Borg G. Escalas de Borg para a Dor e o Esforço Percebido. São Paulo: Manole; 2000.

24. Robertson, R. J., Goss, F. L., Rutkowski, J., Lenz, B., Dixon, C., Timmer, J., Frazee, K., Dube, J., \& Andreacci, J. Concurrent validation of the OMNI perceived exertion scale for resistance exercise. Medicine and Science in Sports and Exercise, 2003, 35(2), 333-341.

25. Colado JC, Furtado GE, Teixeira AM, Flandez J, Naclerio F. Concurrent and Construct Validation of a New Scale for Rating Perceived Exertion during Elastic Resistance Training in The Elderly. J Sports Sci Med. 2020;19(1):175-186.

26. Tiggemann, CL; Pinto, RS; Kruel, LFM. A percepção de esforço no treinamento de força. Revista brasileira de medicina do esporte, São Paulo: SBME, 2010, Vol. 16, n. 4, p. 301-309, 2010.

27. Nasser, I; Neto, VGC. Treinamento de força com baixas cargas e alto volume para hipertrofia: análise de parâmetros moleculares. Revista Brasileira de Prescrição e Fisiologia do Exercício (RBPFEX), 2017, v. 11, n. 68, p. 610-619.

28. Ahtiainen JP, Pakarinen A, Kraemer WJ, Häkkinen K. Acute hormonal and neuromuscular responses and recovery to forced vs. maximum repetitions multiple resistance exercises. International journal of sports medicine. 2003 Aug; 24(06):410-418.

29. Loenneke, J. P., Wilson, J. M., Marín, P. J., Zourdos, M.C., \& Bemben, M. G. Low intensity blood flow restriction training: A meta-analysis.European Journal of Applied Physiology, 2012, 112(5), 1849-1859.

30. Cesar, M. C., Sindorf, M. G., Simoes, R. A., Gonelli, P. G., Montebelo, M. L., \& Pellegrinotti, I. L. Comparison of energy cost of maximal strength and local muscle endurance training in young women. Motri. Vila Real, 2013, v. 9, n. 1, p. 50-55.

31. Smilios I, Pilianidis T, Karamouzis M, Tokmakidis SP. Hormonal responses after various resistance exercise protocols. Medicine \& Science in Sports \& Exercise, 2003, v. 35, n. 4, p. 644-654.

32. Tavares, LD; Ugrinowitsch, C; Silva, CMP; Souza, EOD; Laurentino, GC; Pinheiro, FA; Tricoli, V. Efeito da ordem dos exercícios de força sobre o volume, o lactato e o desempenho. Revista Brasileira de Medicina do Esporte, 2017, 23(3), 194-199. 
33. Fleck, S.J.; Kraemer, W.J. Fundamentos do Treinamento de Força Muscular: Princípios Básicos do Treinamento de Força Muscular. Porto Alegre. Editora Artmed. 2006.

34. Pinto, R. S., Lupi, R., \& Brentano, M. A. Respostas metabólicas ao treinamento de força: Uma ênfase no dispêndio energético. Revista Brasileira de Cineantropometria e Desempenho Humano, 2011, 13(2), 150-157.

35. Alves, RC; Enes, AA; Del Vecchio, FB; Prestes, J; Souza Junior, TP. Efeito do intervalo de recuperação no treinamento de força sobre respostas hemodinâmicas de homens treinados. ConScientiae Saúde, 2019, 18(2), 273.

36. Teixeira, CVLS. Métodos avançados de treinamento para hipertrofia. $2^{\mathrm{a}}$ edição. Create Space, 2015.

37. Marshall PW, Robbins DA, Wrightson AW, Siegler JC. Acute neuromuscular and fatigue responses to the rest-pause method. Journal of science and medicine in sport, 2012;15(2):153-158.

38. Angleri V, Ugrinowitsch C, Libardi CA. Crescent pyramid and drop-set systems do not promote greater strength gains, muscle hypertrophy, and changes on muscle architecture compared with traditional resistance training in well-trained men. European journal of applied physiology. 2017;117(2):359-369.

Conflito de interesses: $\mathrm{O}$ autor desse estudo não tem conflitos de interesse na possível publicação dos resultados apresentados.

Agradecimentos: As voluntárias pelo comprometimento em todas as analises realizadas e ao centro de treinamento DKG. 


\title{
Respostas metabólicas e fisiológicas de três métodos de treinamento de força em mulheres treinadas
}

\author{
Tenerife Daniel $^{1}$, Arakaki Nicollas ${ }^{1}$, Santos Diego ${ }^{1}$ Emmanuel de Medeiros Lima Leonardo $^{2}$, Miguel \\ Enrique $^{3}$, Marsili Guedes Krom ${ }^{3}$, Correia Rocha Alexandre ${ }^{5}$, Guedes Jr Dilmar ${ }^{6}$, Pereira da Silva \\ Rodrigo ${ }^{7}$.
}

\author{
${ }^{1}$ Universidade Metropolitana de Santos \\ ${ }^{2}$ Univeridade Metodista de Piracicaba \\ ${ }^{3}$ Centro Universitário de Espirito Santo do Pinhal \\ ${ }^{4}$ Universidade Metropolitana de Santos, Universidade Federal de São Paulo \\ ${ }^{5}$ Universidade Metropolitana de Santos, Universidade Paulista \\ ${ }^{6}$ Universidade Metropolitana de Santos, Centro de Estudos de Fisiologia do Exercício e Treinamento, \\ Universidade Santa Cecilia \\ ${ }^{7}$ Universidade Metropolitana de Santos, Faculdade Praia Grande, Universidade Federal de São Paulo
}

\begin{abstract}
Resumo
Objetivo: Analisar as respostas metabólicas e fisiológicas dos métodos repetição regressiva alternada, série fixa bilateral e série fixa unilateral em mulheres treinadas.

Metodologia: A amostra foi composta por 10 indivíduos do sexo feminino com média de idade de 32,1 $(3,7)$ anos, praticantes de treinamento de força a mais de 4 anos. Cada voluntária compareceu 4 dias ao laboratório, o primeiro dia para realizar o teste de $1 \mathrm{RM}$ e os demais dias para a realização dos métodos repetição regressiva alternada (RRA), série fixa bilateral (SFB) e série fixa unilateral (SFU) de forma aleatória. Foram aferidas o tempo total, as repetições executadas, o lactato sanguíneo, a frequência cardíaca máxima, a percepção de dor e esforço através das escalas de Borg e Omni.

Resultados: Houve diferença significativa $(\mathrm{p}<0,01)$ nas variáveis repetições entre os métodos: SFB - 41,4 \pm 9,40; SFU - 79,1 \pm 13,3 e RRA - 72,0 \pm 0,0. E tempo total, RRA com 114 segundos, 77 e 150 segundos de diferença para o SFB e SFU respectivamente. Nas outras analises não foi observada diferença significativa.

Conclusão: Não foram encontradas diferença significativas no lactato sanguíneo, volume total, escalas de Borg e Omni entre os métodos. Entretanto, houve diferença significativa no variável tempo que foi menor para o método Repetição Regressiva Alternada em relação aos demais.
\end{abstract}

Palavras chaves: Treinamento de Força, Lactato, Métodos de Treinamento. 